\title{
Complementary and alternative medicine (CAM) use by african american (AA) and caucasian american (CA) older adults in a rural setting: a descriptive, comparative study Norma Cuellar*1, Teresa Aycock², Bridgett Cahill ${ }^{3}$ and Julie Ford 4
}

\author{
Address: ${ }^{1}$ School of Nursing, University of Pennsylvania, Philadelphia, PA, USA, ${ }^{2}$ Regency Hospital, Meridian, MS, USA, ${ }^{3}$ Emergency Department, \\ South Central Regional Medical Center, Laurel, MS, USA and ${ }^{4}$ Hattiesburg VA Clinic, Hattiesburg, MS, USA \\ Email: Norma Cuellar* ncuellar@nursing.upenn.edu; Teresa Aycock - tmom99@aol.com; Bridgett Cahill - mbcahill1@megagate.com; \\ Julie Ford - jfx2@megagate.com \\ * Corresponding author
}

Published: 18 November 2003

BMC Complementary and Alternative Medicine 2003, 3:8

This article is available from: http://www.biomedcentral.com/ /472-6882/3/8

(C) 2003 Cuellar et al; licensee BioMed Central Ltd. This is an Open Access article: verbatim copying and redistribution of this article are permitted in all media for any purpose, provided this notice is preserved along with the article's original URL.
Received: 07 August 2003

Accepted: 18 November 2003

\begin{abstract}
Background: The use of CAM is at an all time high. There is very little research that compares the use of CAM in elders by ethnicity in rural settings. The purpose of the study was to determine if there was a difference between African American and Caucasian American rural elders on use of CAM and self-reported satisfaction with CAM.
\end{abstract}

Methods: The design was a descriptive, comparative study of 183 elders who reported the number of CAM used and satisfaction with CAM. A convenience sample was recruited through community service organizations in the state of Mississippi. The availability of elders through the support groups, sampling bias, subject effect, and self-report were limitations of the study.

Results: The commonest examples of CAM used by rural elders were prayer, vitamins, exercise, meditation, herbs, chiropractic medicine, glucosamine, and music therapy. Significant findings on SES and marital status were calculated. Differences on ethnicity and demographic variables were significant for age, education, and the use of glucosamine.

Conclusions: Health care providers must be aware that elders are using CAM and are satisfied with their use. Identifying different uses of CAM by ethnicity is important for health care practitioners, impacting how health care is provided.

\section{Background}

An increasing number of people are using complementary and alternative medicine (CAM). According to recent studies, $42.1 \%$ of the American population uses some form of CAM, with $39 \%$ of the older population using CAM [1,2]. In 1997, total spending on CAM was estimated at $\$ 32.7$ billion dollars, up from $\$ 22.6$ billion in
1990, a substantial increase that indicates an escalating portion of the population is seeking CAM [2]. Patients may choose to use CAM as a substitute or in conjunction with conventional medicine for a variety of reasons, including 1) dissatisfaction with health care providers and medical outcomes, 2) side effects of drugs or treatments, 3) high health costs (specifically medications), 4) lack of 
control in their own health care practices, and 5) impersonal and technological health care [3-5].

In reviewing the literature, research studies have not reported on CAM use among rural residents, older adults in culturally diverse groups. In rural settings, limited access to medical care often leads to late diagnosis, postponement of treatment, and greater impairments [6]. The older population is a group that has more chronic illnesses, takes longer to recover when sick, and often needs more health care services than their younger cohorts [7]. This may result in CAM use, often influenced by folklore and cultural beliefs. Understanding choices of CAM use is critical to provide optimal care to older, rural patients as certain remedies may be harmful or interfere with conventional medicine.

With the increase in the older population and the number of persons who are choosing CAM, there has been a demand for research to examine the feasibility, benefits, clinical usefulness and development of CAM interventions in older adults. A large proportion of older adults are interested in learning more about CAM and the benefits to health [3]. However, there is very little research that describes CAM use in minority older adults. Therefore, the purpose of this study was to compare older African Americans (AA) and Caucasian Americans (CA) over the age of 50 on 1) use of CAM and 2) self-reported overall satisfaction with CAM being used.

\section{Rural Health and CAM}

Patients in rural areas experience a variety of unmet needs partly due to limited access to primary care, fewer resources to choose from, lower income, less comprehensive health coverage, ill-equipped or poorly staffed health care agencies, and geographic isolation [8]. Rural health care providers often have difficulty in delivering services that target persons with special health care needs, like older adults [9]. Poverty is more widespread in rural areas and even higher among rural minorities, with $35.2 \%$ of rural AA living in poverty compared to $26.9 \%$ of urban AA. Private health insurance coverage is more common for residents of urban areas while Medicare spends more per capita on urban beneficiaries $(\$ 5,288)$ than rural beneficiaries $(\$ 4,375)[6]$. All of these factors may contribute to the use of complementary and alternative therapies that may not be widely accepted in conventional medicine. An estimated $29.5 \%$ of community dwelling older adults use at least one form of CAM with women more likely than men to use CAM [10].

\section{Older adults and CAM}

By the year 2030, older adults will make up $22 \%$ of the total population [7]. Because of a predicted increase in chronic conditions, older adults may be choosing to use
CAM more often than previously to help manage their health. There is lack of information regarding specific costs, benefits, risks, or precautions pertinent to the older adult. Few CAM therapies have federal regulations to guide choices made about CAM.

The most commonly used CAM by the older adult has been reported as chiropractic medicine, herbal remedies, relaxation techniques, megavitamins and religious or spiritual healing [11]. Several reports describe clinically significant interactions between herbals/supplements and prescription medications $[1,12]$. There is a lack of studies related to appropriate dosage and mechanisms of CAM practices in older adults [13]. The use of herbal remedies (ginkgo biloba and ginseng), vitamins, music therapy, touch, massage therapy, and neurofeedback have benefit in the older adult with implications for improved cognitive function [14]. The demographic characteristics that predict CAM use are gender (females use more CAM then men) and education (the higher educated use CAM more often) [5].

In a recent study describing members of Shield 65, a Blue Shield Medicare supplement that offers CAM coverage for people over $65,41 \%$ of older adults used some form of CAM, with $80 \%$ reporting some improvement in their health conditions. Of the older adults who did use CAM, $58 \%$ reported they did not discuss CAM use with their medical doctor or health care practitioner [5].

In a study examining use of CAM in the older adult, more women and fewer African Americans and Hispanics were represented in the sample. These older adults who use CAM cited arthritis, back pain, heart disease, allergies, and diabetes as reasons using CAM [11].

\section{Cultural Diversity and CAM}

Cultural diversity and the health care practices specific to a culture can shape the system of health care in a country. The assumption that conventional medical practice is the choice for all races is incorrect. CAM health care practices in the United States have broadened due to an influx of cultures, values, and beliefs $[4,15]$. Exposure of U.S. citizens to other cultures and cultural healing methods, as well as documented effectiveness of CAM used in different cultures, has spawned interest in CAM in this country. There is little research addressing CAM modalities with origins in racial healing practices and folklore. Most of the CAM surveys include middle class, Caucasian, educated subjects, excluding how race may influence CAM use. The inclusion of folk remedies is often ignored or not discussed. The findings of one study on ethnic minority and CAM use showed no differences between ethnicities but recognized the need to consider CAM practices separately to get an accurate picture of ethnic minority use [16]. 
Race has been reported to affect the choices of CAM [17]. Older women in all cultural groups have expressed more satisfaction with use of CAM than younger women $[10,18,19]$. It is estimated that $83 \%$ of minority patients who use CAM do not report it to their physician [19].

There is a gap in the literature on CAM use by older adults of different racial backgrounds, specifically in rural areas. This survey was undertaken to provide preliminary information for future research on the measurements of outcomes and evidenced based practice in relation to CAM use in older adults of different races. The research questions were:

- Is there a difference in CAM use between AA and CA older adults?

- Is there a difference in satisfaction of CAM use between $\mathrm{AA}$ and CA older adults?

- Overall, what CAM modalities are being used by rural older adults?

\section{Definition of Terms}

The following terms were operationally defined for the survey:

- Complementary and Alternative Medicine (CAM): for the purpose of this study, CAM, as defined by the National Center for Complementary and Alternative Medicine (NCCAM), "is a group of diverse medical and health care systems, practices, and products that are not presently considered to be part of conventional medicine." [20].

- CAM modality use: for the purpose of this study, CAM use was identified in demographic data as a self-report. CAM modality use was presented as a list based on five modalities of NCCAM [20] and the top CAM use reported in the literature. Participants were asked to circle which CAM they used, as many as they used. An open ended option of "other" was provided for CAM not listed (see Table 2 for list). Participants could circle the use of herbs or vitamins, but did not specify which herbs or vitamins were being used.

Table 2: CAM use by AA and CA Rural Older adults

\begin{tabular}{|c|c|c|}
\hline CAM Used & African Americans $(n=40)$ & Caucasian Americans $(n=143)$ \\
\hline Acupuncture & 0 & $\mathrm{I}(.7 \%)$ \\
\hline Aroma Therapy & I (2.5\%) & $3(2.1 \%)$ \\
\hline Art Therapy & I (2.5\%) & I (.7\%) \\
\hline Biofeedback & $2(5 \%)$ & 0 \\
\hline Chelation & 0 & $\mathrm{I}(.7 \%)$ \\
\hline Chiropractic Medicine & $3(7.5 \%)$ & 27 (19\%) \\
\hline Chondroiton & 0 & $13(9.2 \%)$ \\
\hline Exercise & $23(57.5 \%)$ & $94(66.2 \%)$ \\
\hline *Glucosamine & 0 & $28(19.7 \%)$ \\
\hline Herbs & $10(25 \%)$ & $33(23.2 \%)$ \\
\hline Hypnosis & 0 & $\mathrm{I}(.7 \%)$ \\
\hline Journal Writing & $2(5 \%)$ & $12(8.5 \%)$ \\
\hline Magnetic Therapy & $2(5 \%)$ & $9(6.3 \%)$ \\
\hline Massage Therapy & $4(10 \%)$ & $16(11.3 \%)$ \\
\hline Meditation & $12(30 \%)$ & $32(22.5 \%)$ \\
\hline Melatonin & 0 & $7(4.9 \%)$ \\
\hline Metal Therapy & 0 & $2(1.4 \%)$ \\
\hline Music Therapy & $6(15 \%)$ & $15(10.6 \%)$ \\
\hline Naturopathic Medicine & 0 & $4(2.8 \%)$ \\
\hline Prayer & $36(90 \%)$ & $119(83.8 \%)$ \\
\hline Qi & I (2.5\%) & $\mathrm{I}(.7 \%)$ \\
\hline Reiki & 0 & $\mathrm{I}(.7 \%)$ \\
\hline Taichi & 0 & $2(1.4 \%)$ \\
\hline Therapeutic Touch & $2(5 \%)$ & $\mathrm{I}(.7 \%)$ \\
\hline Vitamins & $29(72.5)$ & $122(85.9 \%)$ \\
\hline Visual Imagery & $2(5 \%)$ & $4(2.8 \%)$ \\
\hline Yoga & I (2.5\%) & $4(2.8 \%)$ \\
\hline Other & 0 & $9(6.3 \%)$ \\
\hline
\end{tabular}

*Significant difference by race at $\mathrm{p}=.05$ 
- Satisfaction with CAM: for the purpose of this study, an overall level of satisfaction with CAM modality (or modalities) being used was reported as satisfaction with CAM. There were no measurements for satisfaction of each specific CAM the older adults used.

\section{Methods \\ Sample and Setting}

The survey design was descriptive, comparative, and crosssectional.

A convenience sample of rural AA and CA older adults (over 50 years old) was recruited from Gulfport, Biloxi, Laurel, Hattiesburg, Natchez, Jackson, and Meridian during 10 community service organization meetings in the state of Mississippi, including American Association of Retired Persons, the retired employees of the Southern Pine Electric Power Association known as the Golden Pine Cones Club, and the Retired Seniors Volunteer Group from the local community hospital.

Limitations of the study included the convenience sampling of older adults through these support groups and those who attended, sampling bias, subject effect, and self-report. The advertised topic of the meetings might attract certain people who use CAM than others. The Institutional Review Board (IRB) approval for human subjects protection was obtained at the University of Southern Mississippi, Hattiesburg, MS.

\section{Instruments}

Demographic data included age, gender, race, marital status, socioeconomic status (SES), education, and out of pocket expenses spent on CAM. CAM use in the older adult's health care practices was measured using the five modalities identified by the National Center of Complementary and Alternative Medicine (alternative medical systems, mind body interventions, biologically based therapies, manipulative/body based methods, and energy therapies). Based on the review of the literature and the most often used CAM, a list was provided for the participants to circle the CAM used. An option was given as "other" to assess any CAM use not listed. An overall rating of satisfaction with the CAM used was measured with a Likert scale (1-11). The higher the score, the more satisfied with the CAM. The survey was developed for the study, a limitation of the study.

\section{Procedure}

Community agencies for older adults were contacted throughout the state to ask for participation in the project. At the beginning of the support group meetings, a total of 378 participants were asked to indicate whether or not they had used CAM. This was done by asking if the participants were currently using anything for their own health that was not prescribed by their family physician. Participants who indicated that they had used CAM were then invited to participate in the survey.

An educational program about CAM use was presented after the questionnaires were answered entitled, "What Everyone Should Know About CAM". The program was presented after the collection of data. Topics that were discussed included a brief overview of the following topics: 1) definition of CAM, 2) history of CAM, 3) choosing to use CAM, 4) safety and effectiveness of CAM, 5) contraindications in CAM, 6) choosing a practitioner in CAM, 7) cost, and 8) consulting a health care provider. Information was presented in an unbiased format (neither supportive or opposed to CAM) with the goal to provide general information on CAM to the older adults.

Participation was strictly voluntary and written consent was obtained. Confidentiality was assured by assigning identification numbers matching the consent form with the questionnaire. If the older adult agreed to be in the study, the survey was filled out at the beginning of the meeting. There was no penalty if anyone decided not to participate in the research project. All were invited to stay for the presentation, whether they did or did not participate in the study. After the older adults had answered the questionnaire, the educational presentation was started.

\section{Results}

Data was collected on the older adults who chose to participate and agreed to answer the questionnaire. Of the 378 support group attendees, 183 indicated that they had used CAM. All of these 183 volunteered to participate in the survey and returned completed questionnaires. The sample consisted of $40 \mathrm{AA}$ and $143 \mathrm{CA}$ older adults who volunteered to be participants. Demographic data were collected on the older adults' who agreed to complete the questionnaire on age, gender, race, marital status, SES, education, and out of pocket expenses spent on CAM (Table 1). Significant overall differences between the AA and CA were found on SES $(\mathrm{P}=.008, \mathrm{~F}=3.049, \mathrm{df}=6)$ and marital status $(\mathrm{p}=.042, \mathrm{~F}=4.201, \mathrm{df}=1)$. In the SES ranking, only $13 \mathrm{CA}(14 \%)$ reported an income of $\$ 40,000$ or greater with 13 CA older adults $(11 \%)$ compared to only one AA (3\%). A higher percentage of older AA adults were single $(62.5 \%)$ compared to CA (57\%).

To answer Research Question 1 (Is there a difference in CAM use between AA and CA older adults?), participants were asked to circle the CAM they used on a list provided. The mean number of CAM used by the participants who answered the questionnaire was $3.8(\mathrm{SD}=2.14)$. The range of number of CAM used by each participant was 112. Overall, CA used more CAM products than AA with CA using an average of 4 CAM products and AA using 3 
Table I: Demographics of older adults who reported use of CAM

\begin{tabular}{|c|c|c|c|c|}
\hline Demographic Variable & Intervals & Overall & $\mathrm{AA} n=40$ & $C A n=143$ \\
\hline \multirow[t]{4}{*}{ *Age } & $50-59$ & $7(3.8 \%)$ & 0 & $7(5 \%)$ \\
\hline & $60-79$ & $52(28 \%)$ & 19 (47.5\%) & $33(23 \%)$ \\
\hline & 70-79 & 75 (40\%) & 19 (47.5\%) & 55 (38\%) \\
\hline & $80>$ & $52(28 \%)$ & $2(5 \%)$ & $48(34 \%)$ \\
\hline \multirow[t]{2}{*}{ Gender } & Male & 45 (24\%) & $9(23 \%)$ & $33(23 \%)$ \\
\hline & Female & $139(76 \%)$ & $30(77 \%)$ & $109(77 \%)$ \\
\hline \multirow[t]{2}{*}{ Marital Status } & Married & $78(42 \%)$ & $15(37.5 \%)$ & $61(43 \%)$ \\
\hline & Single & $107(58 \%)$ & $25(62.5 \%)$ & 81 (57\%) \\
\hline \multirow[t]{4}{*}{ Socioeconomic Status (SES) } & $\$ 0-\$ 19,999$ & $95(51.1 \%)$ & $28(72 \%)$ & $64(49 \%)$ \\
\hline & $\$ 20-\$ 39,999$ & $64(34.4 \%)$ & $10(25 \%)$ & $54(4 \mid \%)$ \\
\hline & $\$ 40-\$ 59,999$ & $8(4.4 \%)$ & 0 & $8(6 \%)$ \\
\hline & $\$ 60,000$ and $>$ & $6(3.2 \%)$ & I (3\%) & $5(4 \%)$ \\
\hline *Education & $<8^{\text {th }}$ grade & $16(8.7 \%)$ & $8(21 \%)$ & 7 (5\%) \\
\hline $9-12$ grade & $106(57.6 \%)$ & $25(64 \%)$ & $79(55.5 \%)$ & \\
\hline College & $57(30.6 \%)$ & $5(13 \%)$ & $52(36.5 \%)$ & \\
\hline Graduate School & $5(2.7 \%)$ & $\mathrm{I}(2 \%)$ & $4(3 \%)$ & \\
\hline \multirow[t]{6}{*}{ Out of pocket expenses spent on CAM } & Under $\$ 100.00$ & $84(48.8 \%)$ & $22(55 \%)$ & $60(47 \%)$ \\
\hline & $\$ 101.00-\$ 500.00$ & $56(32.6 \%)$ & 7 (I7.5\%) & $48(37 \%)$ \\
\hline & $\$ 501.00-\$ 1,000$ & $17(9.9 \%)$ & $5(12.5 \%)$ & $12(9 \%)$ \\
\hline & $\$ 1001.00-\$ 1500$ & $7(4.1 \%)$ & $3(7.5 \%)$ & $4(3 \%)$ \\
\hline & $\$ 1501.00-\$ 2000$ & $5(2.9 \%)$ & $2(5 \%)$ & $3(2.5 \%)$ \\
\hline & $\$ 2000$ and more & $3(1.7 \%)$ & $\mathrm{I}(2.5 \%)$ & $2(1.5 \%)$ \\
\hline
\end{tabular}

*Significant difference by race at $\mathrm{p}=.05$

CAM products per person. AA did not use glucosamine as often as CA ( $\mathrm{p}=.002)$. CAM use between AA and CA is seen in Table 2.

Chi square analysis was calculated on race and the demographic variables on CAM use, with significant findings for age $(p=.003)$ and education $(p<.001)$. AA in the group were older and less educated than CA. CAM use was more prevalent in the 50-60 year old age group for the CA $(\mathrm{n}=7)$ than for the AA $(\mathrm{n}=0)$. A significant difference was seen with education with $40 \%$ of CA $(n=56)$ having high school education and higher compared to only $15 \%$ ( $n=$ 6) for AA. The actual CAM use of those who did not respond could modify the estimate of CAM use among the sample attending the workshops.

To answer Research Question 2 (Is there a difference in satisfaction of CAM use between AA and CA older adults?), participants were asked to rate their satisfaction on a Likert scale of $1-11,11$ being the most satisfied with CAM. Using t-test analysis, no differences existed between satisfaction with CAM use. AA mean satisfaction was 8.34 $(\mathrm{SD}=2.25)$ and $\mathrm{CA}$ mean satisfaction was $8.32(\mathrm{SD}=$ 2.25). To compare the AA and CA rural older adults, bivariate correlations were calculated by pairs using Pearson's product moment correlations with $\mathrm{r} 2$ at .08 and .16 for AA and CA, respectively. No correlations existed between number of CAM used and satisfaction with CAM.
To answer Research Question 3 (Overall, what CAM modalities are being used by rural older adults?), number of CAM used was calculated. The most commonly used CAM reported were prayer $(n=155)$, vitamins $(n=151)$, exercise $(n=117)$, meditation $(n=44)$, herbs $(n=43)$, chiropractic medicine $(n=30)$, glucosamine $(n=28)$, and music therapy $(\mathrm{n}=21)$.

Overall 378 older adults were invited to answer the questionnaire for the research project. It is not certain if the 195 persons who did not complete the questionnaire (51\%) who attended the support group meetings did or did not use CAM. Demographic data and information related to CAM use is not known on those who chose not to answer the questionnaire.

In summary, the study finds that rural older adults who used CAM reported satisfaction with use. Differences in CAM use was identified by age and education. CA use more CAM per person than AA with significant findings in the use of glucosamine by CA.

\section{Discussion}

This study is a survey, therefore generalizability of findings should be considered. Differences between CA and AA were found in age and education. AA using CAM were older than the CA. This may be due to the generation of older adults who had to rely more on folklore practices in 
their lives from the early 1900's, when health care access was not available to rural residents or minorities. Very few of these AA were educated past high school, while a larger number of CA were educated at the college level and higher. The AA may have had difficulty in comprehending the questions and may have been less willing to participate in research. Race may also have played a factor in the low response from AA due to mistrust of research activities the AA culture has experienced.

The top five uses of CAM in both AA and CA were prayer, vitamins, exercise, meditation, and herbs. The most common uses of CAM in previous studies identified were chiropractic medicine, herbal remedies, relaxation techniques, megavitamins and religious or spiritual healing [11]. Since Mississippi is considered the "Bible Belt" of the South, the commitment to spirituality may have contributed to the use of prayer as the highest CAM used in older adults. Prayer may also be used more frequently in the elderly who are facing issues related to chronic health conditions, socio-economic status, sociocultural limitations, and end of life decisions. It should be noted that the differences found in the use of vitamins (.06) and chiropractic medicine (.08) were near significance at the .05 level by race; perhaps, with a larger sample size, significant difference may have been obtained. These findings may be due to the lack of insurance that poverty stricken minority older adults face in rural areas like Mississippi. Chiropractic services are often covered by health insurance in this country with a higher likelihood for middle class citizens to be covered by such insurance. There was no difference in the satisfaction of the CAM used by CA or AA. Both groups were satisfied with the CAM being used.

All of the older adults who answered this questionnaire lived in southern Mississippi, considered to be $100 \%$ rural. Mississippi has a high poverty rate with $19.9 \%$ of the population living below poverty, compared to $12.4 \%$ nationwide [21]. Rural older adults may feel the need to use more CAM and folklore practices than conventional medications because of the perceived reliability of these interventions based on folklore or family traditions and the availability of these practices. Of all the CAM, exercise had the highest percentage of usage, excluding nutritional supplements. Being in a rural area, many of the older adults spent their lives doing physical labor, working on farms, and doing blue-collar jobs. This may predispose these older adults to include some form of exercise in their daily lives.

Of the 378 older adults who were asked if they used CAM, $183(49 \%)$ reported CAM use and answered the survey. This number may be an underestimation of the population, as there may have been older adults who used CAM who did not want to participate in the study. However, the number correlates with previously reported results of $41 \%$ to $75 \%$ of older adults using some form of CAM [5]. The majority of the participants were female in a low SES bracket and with no more than a high school education. Many of the participants used a variety of CAM products with an average CAM use of 3.8 interventions per participant. One hundred and fifty five (85\%) participants used prayer, with 151 (83\%) using vitamins, and 117 (64\%) using exercise as CAM interventions. The workshop title may have attracted a certain population more than others. It should also be noted that a number of persons who chose not to participate in the study may use CAM.

Fewer AA attended the community programs than CA. The African American population represents $36.3 \%$ of the Mississippi residents, a high percentage compared to the national average of $12.3 \%$ [21]. AA may avoid these meetings due to feelings of racial discrimination still present between AA and CA in the "deep South". Community churches may be better sources of recruitment for AA.

Despite the fact that almost half of the group who attended the meetings used CAM, only a small percentage of money was being spent by the older adults for CAM. Forty five per cent of the participants reported spending less than $\$ 100 /$ year on CAM. This may be due to the limited income available to spend on CAM (51.1\% of older adults in this study made less than $\$ 19,999 /$ year). Insurance often does not cover CAM so CAM may not be considered due to cost. There may be limited availability of CAM in rural areas indicating that rural residents may have to travel to receive CAM, adding an extra burden of expense.

\section{Conclusions}

Health care providers should be aware of CAM use in older adults, specifically those who live in rural areas who may be familiar with folklore and other alternative interventions in their daily health care practices. Patients in poverty may not have the benefits of expensive CAM interventions, including those reimbursed by health care insurance as many of rural older adults are either uninsured or have limited reimbursement policies through state and government agencies. Health care providers must recognize that CAM may be used very differently among a variety of racial backgrounds. Recommendations for future research may include:

- use of herbs and specific concerns related to aging and metabolism including absorption, distribution, metabolism, and excretion that can affect the interactions of medications among AA and CA elders 
- pharmacological studies specifically for older adults to determine potential interactive effects of CAM between $\mathrm{AA}$ and CA with standard treatment medications, and

- evaluation of the safety and efficacy of CAM practices in AA and CA older adults, specifically herbs and vitamins.

In summary, health care providers must be aware of different uses of CAM by race. Older adults, both AA and CA, may have specific concerns because of gerontological issues that may increase susceptibility to CAM interventions. The responsibility of the health care provider regarding CAM use, side effects, and benefits must be acknowledged. Differences in CAM use by race must also be considered when advising patients.

\section{Competing Interests}

None declared.

\section{Authors' contributions}

NC was the P.I. in this study. She was responsible for designing and coordinating the study including analyzing data, submitting it for publication, as well as presenting findings at international conference.

TA participated in data collection and read and approved of the final manuscript.

BC participated in development of methodology, data collection, and participated in the coordination of the study. She assisted in standardizing the presentation of materials for all the groups as well as developing the program presentation. She edited and approved of the final manuscript.

JF participated in development of methodology, data collection, and participated in the coordination of the study. She assisted in developing the CAM presentation. All authors read and approved of the final manuscript.

\section{Acknowledgements}

This study was funded by the University of Southern Mississippi Research Council and Sigma Theta Tau International Honorary Nursing Society, Gamma Lambda Chapter, Hattiesburg, MS. *Partial support was from grant award T32-AT-00052 CAM Research Training Program funded by the National Center for Complementary and Alternative Medicine, National Institutes of Health.

\section{References}

I. Cherniack EP, Senzel RS and Pan CX: Correlates of use of alternative medicine by the elderly in an urban population. J Altern Complement Med 200I, 7:277-280.

2. Eisenberg DM, Davis RB, Ettner SL, Appel S, Wilkey S, Van Rompay $M$ and Kessler RC: Trends in alternative medicine use in the United States, 1990-1997: results of a follow-up national survey. Jama. I998, 280: I569-I575.

3. Shua-Haim J and Ross J: Alternative medicine in geriatrics: competing with or complementing conventional medicine? Clinical Geriatrics 1999, 7:.
4. Snyder $M$ and Lindquist R: Issues in complementary therapies: how we got to where we are. Online Journal of Issues in Nursing. 200I, 6: I.

5. Astin JA: Why patients use alternative medicine: results of a national study. Jama. 1998, 279:1548-I553.

6. Rural Health Statistics 2001, 2002: [http://www.nal.usda.gov/ric/ richs/stats.htm]. Rural Information Center Health Service, U.S. Department of Agriculture

7. Ebersole $P$ and Hess P: Toward Healthy Aging-Human Needs and Nursing Response. 5thth edition. St. Louis, Mosby; 1998.

8. Cuellar $\mathrm{N}$ and Butts JB: Caregiver distress: what nurses in rural settings can do to help. Nursing Forum 1999, 34:24-30.

9. Improving Health Care for Rural Populations 1996, 2002: [http://www.ahrq.gov/research/rural.htm]. Agency for Health Care Policy and Research

10. Dello Buono M, Urciuoli O, Marietta P, Padoani $W$ and De Leo D: Alternative medicine in a sample of 655 community-dwelling elderly. Journal of Psychosomatic Research. 200 I, 50: I47-I54.

II. Foster DF, Phillips RS, Hamel MB and Eisenberg DM: Alternative medicine use in older Americans. Journal of the American Geriatrics Society. 2000, 48: I560-1565.

12. Kaufman DW, Kelly JP, Rosenberg L, Anderson TE and Mitchell AA: Recent patterns of medication use in the ambulatory adult population of the United States: the Slone survey.[comment]. Jama. 2002, 287:337-344.

13. Luskin FM, Newell KA, Griffith M, Holmes M, Telles S, DiNucci E, Marvasti FF, Hill M, Pelletier KR and Haskell WL: A review of mind/ body therapies in the treatment of musculoskeletal disorders with implications for the elderly. Altern Ther Health Med 2000, 6:46-56.

14. Adams LL, Gatchel RJ and Gentry C: Complementary and alternative medicine: applications and implications for cognitive functioning in elderly populations. Altern Ther Health Med 200I, 7:52-61.

15. Leonard BJ: Quality nursing care celebrates diversity.[comment]. Online Journal of Issues in Nursing. 200I, 6:3.

16. Mackenzie ER, Taylor L, Bloom BS, Hufford DJ and Johnson JC: Ethnic minority use of complementary and alternative medicine (CAM): a national probability survey of CAM utilizers. Altern Ther Health Med 2003, 9:50-56.

17. Jones J: Ethnicity may affect alternative, complementary therapy choices. Journal of the National Cancer Institute. 2001, 93: I522-1523.

18. Cappuccio FP, Duneclift SM, Atkinson RW and Cook DG: Use of alternative medicines in a multi-ethnic population. Ethnicity \& Disease. 200 I, I I: I I- I8.

19. Sleath B, Rubin RH, Campbell W, Gwyther L and Clark T: Ethnicity and physician-older patient communication about alternative therapies. J Altern Complement Med 200I, 7:329-335.

20. What is Complementary and Alternative Medicine (CAM)? 2002, 2002: [http://www.nccam.nih.gov/health/whatiscam/\#supl]. National Center for Complementary and Alternative Medicine

21. FedStats: Mississippi MapStats. 2001, 2003: [http://www.fed stats.gov/qf/states/28000.html].

\section{Pre-publication history}

The pre-publication history for this paper can be accessed here:

http://www.biomedcentral.com/1472-6882/3/8/prepub 